For reprint orders, please contact: reprints@futuremedicine.com

\title{
Systemic therapy for hepatocellular carcinoma
}

Mairéad G McNamara’ \& Jennifer J Knox*1
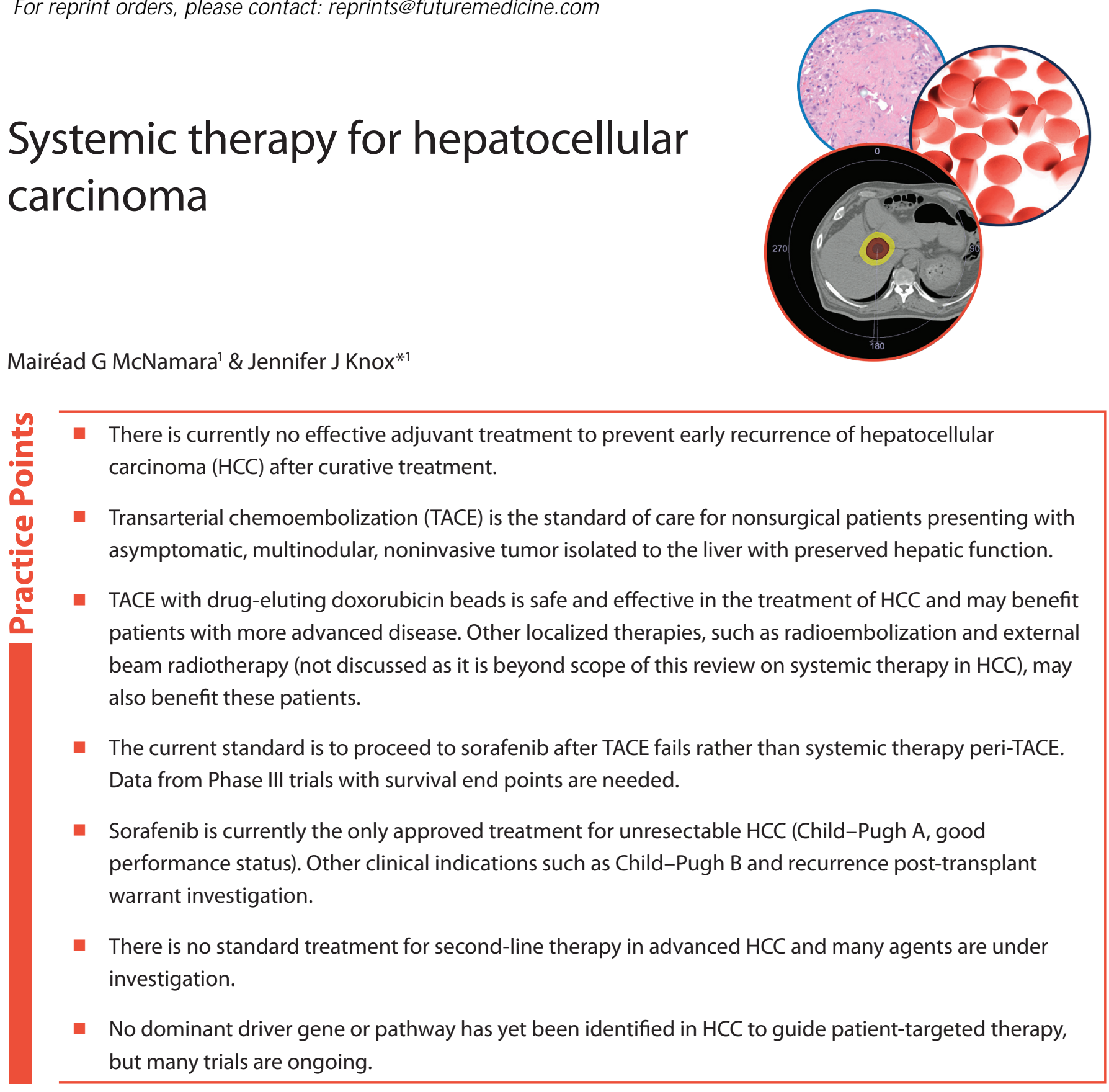

SUMMARY Optimal treatment of hepatocellular carcinoma $(\mathrm{HCC})$ is clinically challenging. Systemic treatment for advanced HCC was limited until the approval of sorafenib. This discovery resulted in the advent of many clinical trials. An ongoing Phase III trial is examining the benefit of adjuvant sorafenib. Utilization of doxorubicin-eluting bead embolization may offer safer treatment in eligible HCC patients. The use of systemic treatment peritransarterial chemoembolization is also being investigated. Many targeted therapies are being explored as first-/second-line treatment options in advanced HCC. The potential benefit of c-MET inhibitors, particularly in those with advanced, MET high expression HCC, may result in new

'Department of Medical Oncology, Princess Margaret Cancer Centre, 610 University Avenue, Toronto, Ontario, M5G 2M9, Canada *Author for correspondence: Tel.: +1 416946 2399; Fax: +1 416946 6546; jennifer.knox@uhn.ca 
systemic patient-directed targeted medicinal approaches. Remaining dilemmas query the appropriate management of patients with advanced Child-Pugh B, HCC and those recurring post-transplant.

Hepatocellular carcinoma (HCC) is the most common liver cancer, accounting for $90 \%$ of primary liver cancers, and is the third leading cause of cancer-related mortality globally, behind lung and stomach cancers [1]. The clinical diagnosis and management of early-stage HCC is improving. However, fewer than $30 \%$ of patients are amenable to curative treatments, such as resection, liver transplant or radiofrequency ablation, owing to the stage of disease, underlying liver disease or limited resources [2]. Transarterial chemoembolization (TACE) has primarily been found to increase survival in patients without major vascular thrombosis [3]. The prognosis for patients with advanced HCC (unresectable or metastatic disease) is poor. In patients unsuitable for TACE, sorafenib, a multitargeted anti-VEGF receptor (VEGFR) and RAF kinase inhibitor, was the first systemic agent shown in a double-blind, randomized Phase III trial involving patients with advanced HCC and ChildPugh A cirrhosis, to improve median overall survival versus placebo (10.7 vs 7.9 months) [4], and thus established a new standard of care in these patients.

The key signaling pathways that have been implicated in the pathogenesis of HCC include those mediated by EGF, VEGF, PDGF, IGF, the Ras/Raf/MAPK/extracellular signal-regulated kinase, Wnt $/ \beta$-catenin and PI3K and tensin homolog deleted on chromosome 10/ Akt/mTOR [5]. The MET receptor for the HGF has also been implicated in the pathogenesis of HCC [6]. Some molecular-targeted agents simultaneously target more than one molecule and this multiple targeting could enhance these agents therapeutic efficacy (e.g. sorafenib, brivanib, regorafenib and sunitinib). Others act on a single target (e.g., everolimus) (Figure 1). Many recent studies have and are investigating the potential of targeting these pathways (Table 1), therefore the landscape of systemic treatment of HCC is constantly changing. The aim of this review is to provide an overview on the current evidence supporting systemic therapy in the treatment of HCC; adjuvant treatment, peri-TACE in earlier stage disease and as primary treatment in advanced disease. Some ongoing key clinical trials in HCC will also be discussed.

\section{Adjuvant treatment: systemic therapy}

No effective adjuvant treatment to prevent early recurrence of HCC after curative treatment has been described. A meta-analysis, published in 2013, of adjuvant treatment after potentially curative treatment for HCC reported that eight adjuvant modalities were identified from 27 eligible randomized controlled trials conducted predominantly in Asian populations comparing adjuvant with no adjuvant therapy [7]. Seven randomized controlled trials were pooled for analysis of effect of interferon adjuvant therapy on recurrence-free survival (liver disease predisposing risk factor was hepatitis $\mathrm{C}$ in four studies and hepatitis B in three studies) and four trials were pooled to determine effects of adjuvant therapy on overall survival [7]. It was concluded that adjuvant interferon improved both recurrencefree survival (hazard ratio [HR]: 0.75; 95\% CI: 0.61-0.92; $\mathrm{p}=0.006$ ) and overall survival (HR: $0.5295 \%$ CI: $0.38-0.71 ; p<0.0001)$, but it was cautioned that the benefits of using this modality should be weighed against its side effects, which possibly negate its global acceptance in clinical practice. Furthermore, the beneficial effects of adjuvant interferon therapy on recurrence and survival may be attributable to its efficacy in preventing hepatitis and cirrhosis as risk factors for development of HCC. A combination of systemic and transhepatic arterial chemotherapy was not recommended for HCC after potentially curative treatment and is possibly owing to the presence of underlying cirrhosis limiting the capability of remnant liver insulted by initially curative treatment to tolerate any adjuvant cytotoxic therapy [7]. This study also concluded that the benefits of adjuvant vitamin analog therapy; acyclic retinoids (vitamin A ana$\log$ ) and menatetrenone (vitamin K2 analog), as chemoprevention required further examination, as the meta-analysis of recurrence-free and overall survival was performed in five small randomized controlled trials in Japan (four trials had less than 61 patients, one trial included had 548 patients) and may thus have methodological weaknesses [7]. Currently, a Phase III randomized, placebo-controlled trial of sorafenib $400 \mathrm{mg}$ twice daily in the adjuvant treatment of HCC after potentially curative treatment (surgical resection or local ablation) is maturing and 


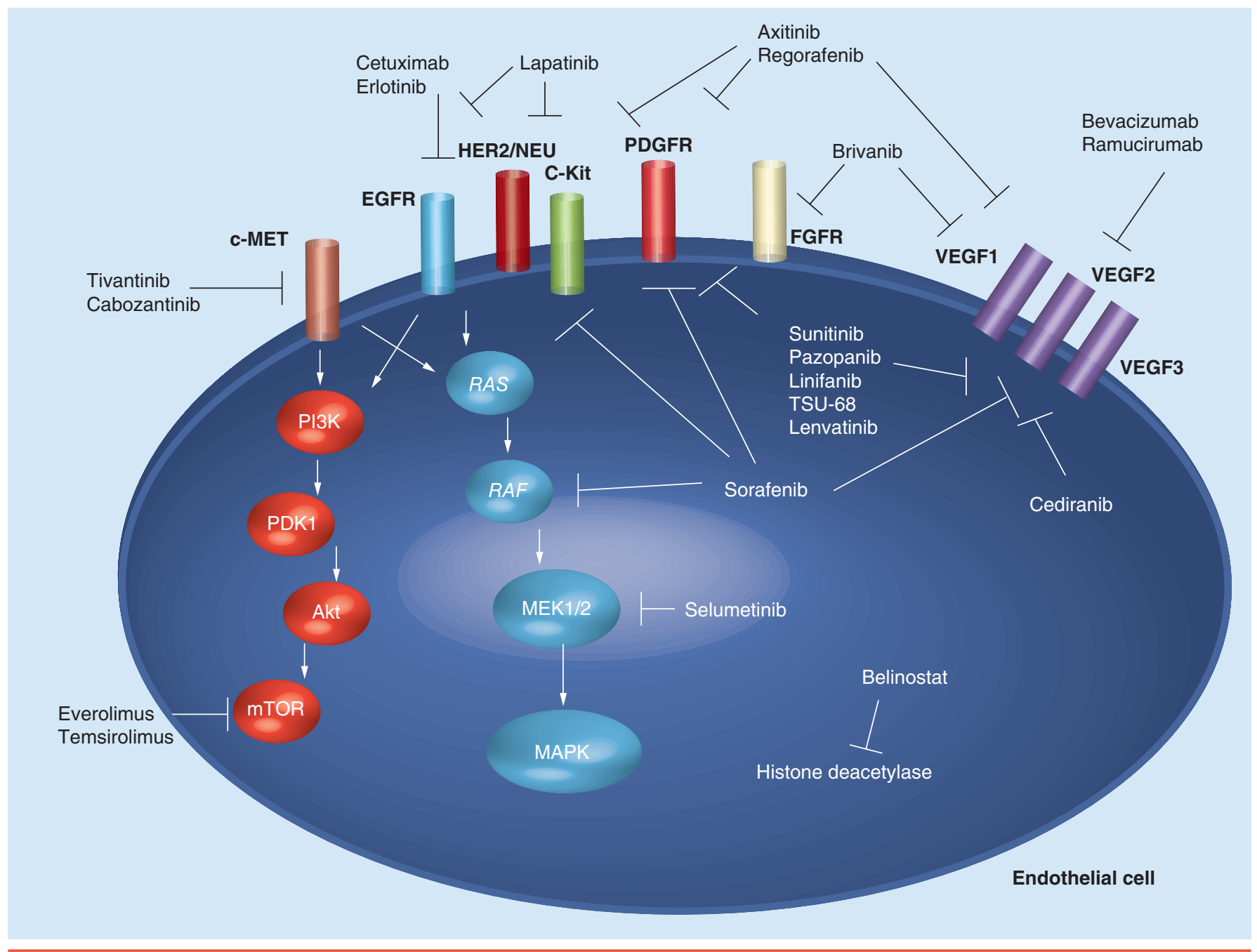

Figure 1. Pathways and targeted agents in the treatment of hepatocellular carcinoma.

EGFR: EGF receptor; FGFR: FGF receptor; PDGFR: PDGF receptor.

we await further direction based on its results [101]. To date, there is no accepted standard of care in the adjuvant setting.

\section{TACE in earlier stage disease}

A meta-analysis published in 2003 reported that TACE was standard of care for nonsurgical patients presenting with asymptomatic, multinodular, noninvasive tumor isolated to the liver with preserved hepatic function [3]. While conventional TACE with administration of a chemotherapeutic agent in oil emulsion followed by embolic agents has been the most utilized technique, the introduction of embolic, drug-eluting beads (enhancing tumor delivery and reducing systemic availability) has provided an alternative to lipiodol-based regimens [8]. The PRECISION V study was a prospective randomized controlled trial comparing conventional TACE with doxorubicin, to TACE with drug-eluting doxorubicin-loaded beads for the treatment of 212 cirrhotic patients with HCC [9]. Patients were stratified according to Child-Pugh status (A/B), Eastern Cooperative Oncology Group (ECOG) performance status $(0 / 1)$, bilobar disease (yes/no), and prior curative treatment (yes/no). It was reported that the drug-eluting bead group showed higher rates of complete response, objective response and disease control compared with the conventional TACE group (27 vs $22 \%, 52$ vs $44 \%$ and 63 vs $52 \%$, respectively). The superiority hypothesis was not met (one sided; $\mathrm{p}=0.11$ ). However, Child-Pugh B patients, those with ECOG performance status 1, bilobar disease and recurrent disease showed a significant increase in 
Table 1. Summary of some of the targeted therapies explored in hepatocellular carcinoma

to date.

\begin{tabular}{|c|c|c|c|}
\hline $\begin{array}{l}\text { Targeted } \\
\text { therapy }\end{array}$ & Molecular target & Study line of treatment & Phase of trial study \\
\hline Sorafenib & $\begin{array}{l}\text { VEGFR2, PDGFR, FLT3, RET, } \\
\text { c-KIT, RAF }\end{array}$ & $\begin{array}{l}\text { Adjuvant } \\
\text { Peri-TACE } \\
\text { First-line advanced }\end{array}$ & $\begin{array}{l}\text { III [101] } \\
\text { III [17] } \\
\text { I [49]; II [14,40]; III }[4,22,102,106]\end{array}$ \\
\hline TSU-68 & VEGFR2, PDGFR, FGFR & $\begin{array}{l}\text { Peri-TACE } \\
\text { First-line advanced }\end{array}$ & $\begin{array}{l}\text { II }[20] \\
\text { I/II }[31]\end{array}$ \\
\hline Bevacizumab & VEGF & $\begin{array}{l}\text { Peri-TACE } \\
\text { First-line advanced } \\
\text { Second-line advanced }\end{array}$ & $\begin{array}{l}\text { II }[19] \\
\text { II }[42-44] \\
\text { II }[60]\end{array}$ \\
\hline Belinostat & Histone deacetylase inhibitor & First-line advanced & $\mathrm{I} / \mathrm{II}[38]$ \\
\hline Cediranib & VEGF & First-line advanced & II [30] \\
\hline Cetuximab & EGFR inhibitor & First-line advanced & II $[34,47,48]$ \\
\hline Linifanib & VEGFR, PDGFR & First-line advanced & III [27] \\
\hline Lapatinib & EGFR1,2, HER2/NEU & First-line advanced & II $[35,36]$ \\
\hline Lenvatinib & $\begin{array}{l}\text { VEGFR1-3, FGFR1-4, RET, KIT, } \\
\text { PDGF } \beta\end{array}$ & First-line advanced & I/II [32]; III [105] \\
\hline Pazopanib & $\begin{array}{l}\text { VEGFR1,2,3, PDGFR, FGFR, } \\
\text { cytokine receptors }\end{array}$ & First-line advanced & $\mathrm{I}[33]$ \\
\hline Ramucirumab & VEGFR2 & First-line advanced & II [29]; III [104] \\
\hline Selumetinib & MEK inhibitor & First-line advanced & II [37] \\
\hline Brivanib & VEGF, FGF & $\begin{array}{l}\text { First-line advanced } \\
\text { Second-line advanced }\end{array}$ & $\begin{array}{l}\text { III [28] } \\
\text { II [52]; III [53] }\end{array}$ \\
\hline Erlotinib & EGFR inhibitor & $\begin{array}{l}\text { First-line advanced } \\
\text { Second-line advanced }\end{array}$ & $\begin{array}{l}\text { II }[42-45] \\
\text { II }[60]\end{array}$ \\
\hline Everolimus & m-TOR inhibitor & $\begin{array}{l}\text { First-line advanced } \\
\text { Second-line advanced }\end{array}$ & $\begin{array}{l}1[39,49] \\
\text { I/II [57]; III [110] }\end{array}$ \\
\hline Sunitinib & VEGFR, PDGF & $\begin{array}{l}\text { First-line advanced } \\
\text { Second-line advanced }\end{array}$ & $\begin{array}{l}\text { III }[24] \\
\text { II [55]; III [23] }\end{array}$ \\
\hline Temsirolimus & m-TOR inhibitor & $\begin{array}{l}\text { First-line advanced } \\
\text { Second-line advanced }\end{array}$ & $\begin{array}{l}\text { II }[46] \\
\text { II }[111]\end{array}$ \\
\hline Axitinib & VEGFR 1, 2, 3, PDGF, c-KIT & Second-line advanced & II $[56,108,109]$ \\
\hline Cabozantinib & c-MET, VEGFR2 inhibitor & Second-line advanced & II [59]; III [113] \\
\hline Regorafenib & $\begin{array}{l}\text { VEGFR1,2,3, PDGFR, FGFR, } \\
\text { c-KIT, RET, B-RAF }\end{array}$ & Second-line advanced & II $[54,107]$ \\
\hline Tivantinib & c-MET inhibitor & Second-line advanced & II [58] \\
\hline
\end{tabular}

objective response $(\mathrm{p}=0.038)$ compared with utilization of conventional TACE. The use of drug-eluting beads was also associated with improved tolerability and a significant reduction in serious liver toxicity $(\mathrm{p}<0.001)$ with a significantly lower rate of doxorubicin-related side effects seen $(\mathrm{p}=0.0001)$.

Stereotactic body radiotherapy (24-54 Gy in six fractions) [10,11] and radioembolization [12] have also been used for the treatment of patients with active HCC unsuitable for standard locoregional therapies, and data have provided rationale for their study in randomized trials [10-12]. A prospective, randomized trial of the addition of sorafenib to yttrium-90 radioembolization as a bridge to transplantation in 15 patients with 16 HCC lesions reported that the addition of sorafenib did not augment radiological or pathological response to yttrium-90 therapy for HCC [13]. Equivalent significant reduction in enhancement at 1 and 3 months by European Association for the Study of the Liver guidelines or modified Response Evaluation Criteria in Solid Tumors (RECIST) was noted and neither the European Association for the Study of the Liver nor modified RECIST could reliably predict complete pathological necrosis [13]. A Phase III trial studying the safety and 
Table 2. Some ongoing Phase III trials of transarterial chemoembolization in hepatocellular carcinoma.

\begin{tabular}{|llllll|}
\hline Location & Study & $\begin{array}{l}\text { Patients } \\
(\mathbf{n})\end{array}$ & $\begin{array}{l}\text { Primary } \\
\text { end point }\end{array}$ & $\begin{array}{l}\text { Estimated } \\
\text { completion }\end{array}$ & Ref. \\
$\begin{array}{l}\text { University College London, } \\
\text { London, UK (TACE-2) }\end{array}$ & $\begin{array}{l}\text { TACE DEB } \pm \text { sorafenib } \\
\text { NCT01324076 }\end{array}$ & 412 & PFS & 2014 & {$[116]$} \\
USA Intergroup (ECOG 1208) & $\begin{array}{l}\text { TACE } \pm \text { sorafenib } \\
\text { NCT01004978 }\end{array}$ & 400 & PFS & 2018 & {$[117]$} \\
$\begin{array}{l}\text { Asia (Taiho Pharmaceutical } \\
\text { company, Tokyo, Japan) }\end{array}$ & TACE \pm orantinib (TSU-68) & 880 & OS & 2017 & {$[118]$} \\
$\begin{array}{l}\text { Bristol-Myers Squibb, NY, USA } \\
\text { (Brisk TA study) }\end{array}$ & TACE \pm adjuvant brivanib & 870 & OS & 2014 & {$[119]$} \\
\hline DEB: Doxorubicin-eluting bead; OS: Overall survival; PFS: Progression-free survival. & & & & \\
\hline
\end{tabular}

effectiveness of intra-arterial TheraSphere ${ }^{\circledR}$ (containing radioactive yttrium-90 [Nordion Canada Inc., Ontario, Canada]) is currently enrolling and will be evaluated in patients with unresectable HCC in whom treatment with standard of care sorafenib is planned. All patients receive the standard of care sorafenib with or without the addition of TheraSphere [102] and results are awaited. However, further details of these modalites are beyond the scope of this review on systemic therapies in HCC.

\section{Systemic therapy peri-TACE}

The use of systemic treatment peri-TACE was investigated in the Phase II randomized SPACE trial [14] (sorafenib or placebo in combination with TACE with drug-eluting doxorubicin beads for intermediate stage HCC - Barcelona Clinic Liver Cancer stage B; comprising patients with asymptomatic multinodular HCC [15]). TACE induces necrosis at the tumor site and may create conditions that permit or encourage angiogenesis [16], so the hypothesis is that treatment with an antiangiogenic agent may curtail the post-TACE rise in VEGF-mediated signaling and prevent or delay tumor progression and, thus, the combination of doxorubicineluting beads and sorafenib may be synergistic. Patients were randomized to receive sorafenib $400 \mathrm{mg}$ twice daily or matching placebo continuously (one cycle: 4 weeks) until progression. All patients received drug-eluting doxorubicin beads (150-mg doxorubicin) 3-7 days after the first dose of study drug, and then on day 1 ( \pm 4 days) of cycles three, seven and 13, and every six cycles thereafter. The primary end point was time to radiologic progression. A total of 307 patients were randomized to sorafenib $(\mathrm{n}=154)$ or placebo $(\mathrm{n}=153)$. Although the study met its primary end point of improving time to radiologic progression when sorafenib was added to a regimen of TACE with drugeluting doxorubicin beads compared with drug-eluting doxorubicin beads alone [14], the difference was small and not thought to be clinically meaningful. The combination was well tolerated and no new safety findings that would preclude use of the combination were observed, although fewer than expected TACE procedures were delivered, possibly owing to toxicity of the combination. Regardless, the efficacy signal requires confirmation with data from ongoing Phase III trials (Table 2), and the current standard outside of a clinical trial is to treat with sorafenib only after TACE fails.

A Phase III study of sorafenib given 1-3 months after TACE in Japanese and Korean patients with unresectable HCC and Child-Pugh A cirrhosis who had $\geq 25 \%$ tumor necrosis/shrinkage post-TACE, found that sorafenib did not significantly prolong time to progression in patients who responded to TACE [17]. The authors concluded that this may have been owing to delays in starting sorafenib after TACE.

A Japanese retrospective study of 51 patients with unresectable HCC investigated the efficacy of TACE using cisplatin as second-line treatment in patients unresponsive to TACE using epirubicin-lipiodol emulsion [18]. Response rate was $11.8 \%$ measured by RECIST. Overall survival rates were 61.9, 48.2 and $28.9 \%$ at 1,2 and 3 years, respectively, and median survival time was 15.4 months. Progression-free survival rate was $35.2 \%$ at 1 year, and median progressionfree survival was 3.1 months. No major complications were observed. It was concluded that switching the TACE drug from epirubicin to cisplatin may be a feasible option for advanced HCC, even when considered resistant to the 
initial form of TACE, but larger studies are warranted before firm conclusions can be drawn.

A Phase II trial was conducted to evaluate the safety and efficacy of bevacizumab combined with chemoembolization in 25 patients with unresectable Child-Pugh A or B HCC. Treatment consisted of bevacizumab every 2 weeks and chemoembolization during week 3 of a 6-week cycle for up to three cycles over 6 months. Concurrent treatment with bevacizumab and chemoembolization was found to be safe in this study and the objective response rate was $60 \%$ using enhancement response evaluation criteria. Disease control rate was $100 \%$ and overall survival was 10.8 months, supporting the potential for further development of bevacizumab combined with chemoembolization as a treatment for unresectable HCC [19].

A randomized multicenter Phase II study of TSU-68 (an oral tyrosine kinase inhibitor of VEGFR2, PDGF receptor [PDGFR] and FGF receptor) in 103 patients with a diagnosis of intermediate-stage HCC suitable for TACE, who had been treated with a single session of TACE received either 200-mg TSU-68 twice daily or no medication. The primary end point was progression-free survival. There was a trend towards prolonged progression-free survival with TSU-68 treatment after a single session of TACE, but this was not statistically significant and there were two deaths related to study treatment in the TSU- 68 group; grade 5 hepatic failure and melena [20].

Advanced HCC: systemic standards of care - Sorafenib

Sorafenib, a multikinase inhibitor with activity against RAF kinase, VEGFR2, PDGFR, FLT3, RET and c-KIT, was approved by the US FDA for the treatment of advanced HCC on the basis of the SHARP Phase III study, where the median survival and the time to radiologic progression was nearly 3 months longer for ChildPugh liver function class A patients treated with sorafenib $400 \mathrm{mg}$ twice daily than patients administered placebo [4]. Subset analyses of this study showed that sorafenib consistently improved median overall survival and disease control rate compared with placebo irrespective of disease etiology, baseline tumor burden, performance status, tumor stage and prior therapy [21]. The Phase III sorafenib Asia-Pacific trial conducted in China, Taiwan and South Korea confirmed in 226 patients with well-preserved liver function ( $>95 \%$ Child-Pugh A), that sorafenib improved overall survival and is safe for patients with advanced HCC and also did so irrespective of baseline status (whether hepatitis B virus was present or absent, tumor burden (macroscopic vascular invasion and/ or extrahepatic spread present/absent), presence or absence of either lung or lymph node metastasis at baseline, ECOG performance status $(0,1-2)$, serum concentrations of alanine aminotransferase/aspartate aminotransferase (normal, mildly elevated, moderately elevated), $\alpha$-fetoprotein (normal/elevated) and total bilirubin (normal/elevated) and whether there was a history of hepatectomy or TACE/embolization) [22]. Both of these studies selected patients with well-preserved liver function (Child-Pugh A). If these trials had included patients with more advanced liver failure (Child-Pugh B or C), deaths related to advanced liver disease may have masked any significant effect of sorafenib. Further data are needed to confirm the safety and survival benefits of sorafenib in patients with poorer liver function.

Despite nearly identical HRs for improvement in overall survival (0.69 in SHARP study vs 0.68 in Asia-Pacific study), the median survival for sorafenib compared with placebo in the SHARP trial was 10.7 months compared with 7.9 months [4], as compared with 6.5 months compared with 4.2 months in the Asia-Pacific study [23]. Explanation of the disparity is not clear and may include differences in disease etiology and geographic variations in patient population. In addition, in the SHARP trial, median overall survival in those patients who were positive for antihepatitis $\mathrm{C}$ virus antibody was 14.0 months versus 9.7 months in those positive for hepatitis B surface antigen, but examination of the subset with hepatitis $\mathrm{B}$-associated HCC showed that $56.3 \%$ of those treated with sorafenib compared with $39.3 \%$ of those who received placebo had an ECOG performance status $>0$, indicating that the sorafenib group was at a more advanced clinical stage [21]. In the Asia-Pacific study, 165 of the 226 patients enrolled were infected with hepatitis B virus and sorafenib enhanced both overall survival and time to progression relative to placebo in patients with hepatitis B-associated HCC, further suggesting that the results observed in the SHARP patients with hepatitis associated HCC were owing to patient imbalance and not to these patients responding differently to sorafenib. 
Including the combined results of these two trials and of Cheng et al. [24], the data support the effectiveness of sorafenib for the treatment of advanced HCC irrespective of viral etiology. The observation of potential improved survival in the hepatitis $\mathrm{C}$ group warrants further study and argues for stratification of viral etiology in future trials.

GIDEON is a surveillance study that aims to evaluate the safety of sorafenib in all patients with unresectable HCC under real-life practice conditions (>3000 patients from 39 countries), particularly Child-Pugh B patients, who have not been well represented in clinical trials to date. The first interim analysis has shown global and regional differences in patient characteristics, disease etiology and practice patterns and does not support the routine use of sorafenib in Child-Pugh B patients [25]. However, it does further validate the benefit of sorafenib in real world patients with Child-Pugh A status, as a median overall survival of 10.3 months was reported similar to the SHARP Phase III data [4]. Another study has reported a median survival of 3.6 months among patients treated with sorafenib in advanced HCC with Child-Pugh B or $\mathrm{C}$ status [26], questioning the clinical benefit in such an end-stage population.

A randomized Phase III study of sorafenib (400 mg twice daily orally on days $1-28$ of a 28 -day cycle) versus stereotactic body radiation therapy followed by sorafenib in locally advanced HCC is currently recruiting participants in the USA and Canada [103]. Patients undergo stereotactic body radiation therapy every $24-72 \mathrm{~h}$ for a total of five fractions over 5-15 days. Within 1-5 days poststereotactic body radiation therapy, patients receive sorafenib $200 \mathrm{mg}$ twice daily orally on days 1-28 and then sorafenib is administered at a dose of $400 \mathrm{mg}$ twice daily orally from cycle two onwards, as tolerated. Sorafenib treatment repeats every 28 days for up to 5 years in the absence of disease progression or unacceptable toxicity. The primary outcome measure is to determine if stereotactic body radiation therapy improves overall survival in HCC patients with locally advanced and often marked macrovascular tumor invasion while treated with sorafenib.

Sorafenib is the only currently approved treatment for unresectable HCC. However, other agents are being investigated for their therapeutic potential in advanced HCC and some will now be discussed.

\section{- Sunitinib}

A Phase III open-label trial of sunitinib (multitargeted receptor tyrosine kinase inhibitor) versus sorafenib in patients with advanced HCC, with Child-Pugh A, liver function who had received no prior systemic treatment, concluded that sunitinib failed to meet its primary overall survival end point, with a strong trend to inferiority with sunitinib. Progression-free survival and time to progression results were similar between the sunitinib and sorafenib groups. In a hepatitis B patient subgroup, no significant difference in overall survival was detected between the sunitinib and sorafenib groups. Sunitinib was generally associated with more frequent toxicities than sorafenib [24].

\section{- Linifanib}

A Phase III global trial of linifanib (a potent and selective inhibitor of VEGF and PDGFR tyrosine kinase families), $17.5 \mathrm{mg}$ once daily versus sorafenib $400 \mathrm{mg}$ twice daily in 1035 patients with advanced Child-Pugh A, HCC resulted in similar overall survival in both groups. Median overall survival was 9.1 months (95\% CI: 8.1-10.2) on linifanib and 9.8 months (95\% CI: 8.3-11.0) on sorafenib. However, predefined superiority and noninferiority overall survival boundaries were not met for linifanib. Secondary end points, time to progression and objective response rate favored linifanib, while safety results favored sorafenib [27].

\section{- Brivanib}

Brivanib, a selective dual inhibitor of VEGF and FGF receptors was compared with sorafenib in the first-line treatment of 1155 patients with unresectable, advanced HCC in a multinational, randomized Phase III trial [28]. The study did not meet its primary end point of noninferiority in overall survival for brivanib compared with sorafenib, since the upper limit of $95 \%$ CI for the HR exceeded the noninferiority margin of 1.08, but did demonstrate anti-tumor activity similar to sorafenib, based on the secondary end points, time to progression and disease control rate, which were similar in both arms.

\section{- Ramucirumab}

A Phase II study of ramucirumab (recombinant human monoclonal antibody that binds to the extracellular domain of the VEGFR2) at $8 \mathrm{mg} / \mathrm{kg}$ intravenously every 2 weeks until disease progression or intolerable toxicity, as 
first-line monotherapy in 42 patients with advanced Child-Pugh A/B cirrhosis conferred moderate disease control in advanced sorafenibnaive HCC and was well tolerated for most patients. Serious adverse events (grade 3 or higher) included fatigue in $10 \%$, gastrointestinal bleeding in $9 \%$, hypertension in $14 \%$, infusionrelated reactions in $5 \%$ and headaches in $2 \%$ of patients [29]. A Phase III multicenter, randomized study of ramucirumab (IMC-1121B) drug product and best supportive care versus placebo and best supportive care as second-line treatment in patients with HCC after first-line therapy with sorafenib (REACH) is ongoing [104].

\section{- Cediranib}

A Phase II study assessed the efficacy and toxicity of cediranib (AZD2171; potent inhibitor of VEGF signaling), $45 \mathrm{mg}$ orally, once daily, in 28 patients with advanced HCC. A total of $93 \%$ of patients experienced a grade $3+$ adverse event (fatigue [46\%], anorexia [25\%], hypertension [21\%] and elevated aminotransferase [18\%]) and owing to toxicity, cediranib at this dose and schedule was concluded not to be an effective treatment in patients with unresectable or metastatic HCC [30].

\section{- TSU-68}

The safety and effectiveness of TSU-68, which inhibits angiogenesis, has also been studied in patients with advanced HCC. A total of 12 patients were enrolled in Phase I, and $200 \mathrm{mg}$ twice daily was established as the Phase II dose. Phase II included an additional 23 patients. One patient had a complete response (2.9\%), two patients (5.7\%) had a partial response and 15 patients (42.8\%) showed stable disease. Median time to progression was 2.1 months and median overall survival was 13.1 months. Common adverse events were hypoalbuminemia, diarrhea, anorexia, abdominal pain, malaise, edema and transaminase elevation [31].

\section{- Lenvatinib}

Lenvatinib is an oral tyrosine kinase inhibitor targeting VEGFR1-3, FGF receptor 1-4, RET, KIT and PDGF receptor- $\beta$. A Phase I/II study of lenvatinib in HCC patients with Child-Pugh $A$ and $B$ status concluded that lenvatinib $12 \mathrm{mg}$ once daily in Child-Pugh A, HCC patients and $8 \mathrm{mg}$ once daily in Child-Pugh B, HCC patients were associated with manageable toxicity and preliminary evidence of anti-tumor activity [32].
A multicenter, randomized, Phase III trial is currently ongoing comparing the efficacy and safety of Lenvatinib (E7080) versus sorafenib in the first-line treatment of Child-Pugh A, unresectable HCC. The primary outcome measure is overall survival [105].

\section{- Pazopanib}

A Phase I dose-escalating study of pazopanib (200-800 mg; inhibitor of VEGFR1-3, PDGFRs, FGF receptors and cytokine receptors), once daily on a 21-day cycle in 28 Asian patients with advanced HCC concluded that the maximum tolerated dose in Child-Pugh A patients was $600 \mathrm{mg}$ once daily and recommended this as the dose to be chosen for further development in advanced HCC. In total, 19 patients (73\%) had either partial response or stable disease [33]. Pazopanib had a manageable safety profile and was also shown to reduce tumor vessel leakage, as shown by dynamic contrast-enhanced MRI, indicating a possible direct effect on HCC vasculature that may be associated with its anti-tumor activity.

\section{- Cetuximab}

A Phase II study of the EGF receptor (EGFR) inhibitor, cetuximab, in 30 patients with unresectable or metastatic HCC was safely administered with tolerable toxicity profiles, but in monotherapy did not demonstrate any anti-tumor activity [34].

\section{- Lapatinib}

Lapatinib is an EGFR1, EGFR2 and HER2/ NEU inhibitor. A Phase II study in 40 patients with advanced HCC revealed a progressionfree survival of 2.3 months (95\% CI: 1.7-5.6) and overall survival of 6.2 months $(95 \% \mathrm{CI}$ : 5.1-not available). EGF genotyping indicated HCC patients with $<20$ repeats had the lowest progression-free survival. Therapy with lapatinib in this study did not meet the predefined efficacy rate [35]. Another multi-institutional Phase II study assessing the efficacy and tolerability of lapatinib in 26 patients with advanced HCC, of which $19 \%$ had prior therapy, did not report any objective responses and a median progressionfree survival of 1.9 months and a median overall survival of 12.6 months was seen [36].

\section{- Selumetinib}

Selumetinib, an orally available inhibitor of MAPK tyrosine kinase activity was administered 
at $100 \mathrm{mg}$ twice daily continuously to 19 patients in a Phase II study, with locally advanced or metastatic HCC (82\% Chid-Pugh A) who had not been treated with prior systemic therapy. No radiological responses were seen and median time to progression was 8 weeks, suggesting minimal single-agent activity [37].

\section{- Belinostat}

Therapy with belinostat, a histone deacetylase inhibitor, has been assessed in patients with unresectable HCC. The Phase I study consisted of 18 patients. The maximum tolerated dose was not reached at $1400 \mathrm{mg} / \mathrm{m}^{2}$ administered once daily on days $1-5$ every 3 weeks This dose was used in Phase II and included 42 patients. The partial response and stable disease rates were 2.4 and $45.2 \%$, respectively. The median progression-free survival and overall survival were 2.64 and 6.6 months, respectively. Exploratory analyses revealed that disease stabilization rate (complete response plus partial response plus stable disease) in tumors having high and low HR23B expression (a potential biomarker for response to belinostat) were 58 and $14 \%$, respectively $(\mathrm{p}=0.036)[38]$.

\section{- Everolimus}

A Phase I study has reported that the maximum tolerated dose of the oral mTOR inhibitor everolimus, given first-line in patients with locally advanced or metastatic HCC (Child-Pugh A or B), is $7.5 \mathrm{mg}$ daily [39]. This study also recommended that prophylactic antiviral therapy should be mandatory for hepatitis B surface antigen seropositive patients.

Despite potential improved affinity for the VEGF pathway and other novel targets to date, none of the above discussed agents have demonstrated superior outcomes to sorafenib in the first-line treatment of advanced HCC.

\section{Combination systemic therapy in advanced HCC}

The concept that combination therapy targeting different signaling pathways may improve outcomes has also been explored and will now be discussed.

\section{Doxorubicin \& sorafenib}

A randomized Phase II multinational study reported that among patients with advanced HCC and Child-Pugh A status, who received treatment with doxorubicin $\left(60 \mathrm{mg} / \mathrm{m}^{2}\right)$ and sorafenib (400 mg twice daily) versus doxorubicin alone, had an improved median time to progression (6.4 vs 2.8 months), progression-free survival ( 6.0 vs 2.7 months) and overall survival (13.7 vs 6.5 months), respectively [40]. This trial has served as the basis for the ongoing Phase III trial being conducted across North America of sorafenib plus doxorubicin versus sorafenib alone in patients with locally advanced or metastatic HCC and no prior systemic therapy [106].

\section{Infusional fluorouracil, leucovorin}

\section{\& oxaliplatin}

A randomized Phase III study in mainland China, Taiwan, Korea and Thailand of 371 patients who had locally advanced or metastatic HCC, ineligible for curative resection or local treatment, were assigned at a ratio of 1:1 to receive either fluorouracil, leucovorin and oxaliplatin (FOLFOX4) or doxorubicin. The study did not meet its primary end point of overall survival. Median overall survival was 6.4 months with FOLFOX4 and 4.97 months with doxorubicin (HR: 0.8; 95\% CI: 0.63-1.02; $\mathrm{p}=0.07$ ). The authors concluded that the trend towards improved overall survival with FOLFOX4, along with increased progression-free survival and response rate suggested that this regimen may confer some benefit to Asian patients, but an overall survival benefit could not be concluded [41].

\section{Bevacizumab \& erlotinib}

In 2009, a Phase II trial of the combination of bevacizumab (monoclonal antibody that binds VEGF) and erlotinib (EGFR inhibitor) in 40 patients who had advanced HCC (Child-Pugh A or B), who had failed one prior systemic therapy, reported a 16-week progressionfree survival of $62.5 \%$ with a median overall survival of 68 weeks (95\% CI: 48-78 weeks) and concluded that this combination warranted additional evaluation in randomized controlled trials [42]. Another Phase II study which was performed to evaluate the efficacy and tolerability of bevacizumab, $10 \mathrm{mg} / \mathrm{kg}$ intravenously every 14 days and erlotinib, $150 \mathrm{mg}$ orally daily in 59 advanced HCC patients reported a median progressionfree survival of 7.2 months (95\% CI: 5.6-8.3) and median overall survival of 13.7 months (95\% CI: 9.6-19.7). Grade 3-4 adverse events included fatigue (30\%), diarrhea (17\%), hypertension (14\%), elevated transaminases (12\%) and gastrointestinal hemorrhage (10\%). High plasma ANG 2, EGFR and ET-1, and lack of acneiform 
rash were associated with poor outcome [43]. A Phase II multicenter study across several Asian countries also examined the bevacizumab/erlotinib combination in 51 advanced Child-Pugh A, HCC patients and reported good tolerability with grade 3/4 toxicities being uncommon, a median progression-free survival of 2.9 months (95\% CI: 1.3-4.4) and median overall survival of 10.7 months (95\% CI: 6.2-15.2 months) [44]. By contrast, another study evaluating the combination of erlotinib and bevacizumab in 21 previously untreated inoperable and metastatic HCC patients concluded that this combination did not appear to have sufficient efficacy in these patients and may not warrant further investigation [45].

Temsirolimus \& bevacizumab

A multicenter Phase II trial of the mTOR inhibitor, temsirolimus (25 mg intravenously day $1,8,15$ and 22 every 28 days) and bevacizumab $(10 \mathrm{mg} / \mathrm{kg}$ intravenously day 1 and 15 every 28 days) in Child-Pugh A patients with advanced HCC (no prior systemic therapy involving the VEGF or mTOR class of agents) closed accrual at the end of stage 1 of a modified two-stage Simon design (25 patients eligible for toxicity and efficacy), as neither number of responses nor the progression-free survival at 6 months passed the futility stopping rule for this combination. Median time to progression on study was 6 months, median progression-free survival was 7.4 months and median survival was 8.3 months [46].

Capecitabine, oxaliplatin \& cetuximab

A Phase II study of capecitabine $\left(850 \mathrm{mg} / \mathrm{m}^{2}\right.$ twice daily, days 1-14; oxaliplatin $130 \mathrm{mg} / \mathrm{m}^{2}$ on day 1$)$, and cetuximab $\left(400 \mathrm{mg} / \mathrm{m}^{2}\right.$ on day 1 , then $250 \mathrm{mg} / \mathrm{m}^{2}$ weekly for each 21-day cycle) in patients who had chemotherapynaive advanced/unresectable HCC and any Child-Pugh class chronic liver disease concluded that time to progression (4.5 months [95\% CI: 3.2-6.4]), and overall survival [4.4 months [95\% CI: 2.4-7.3]) were shorter than would be expected for treatment with sorafenib and although the combination was tolerable, diarrhea was pronounced in this population [47] and, thus, this combination can not be recommended based on available evidence to date.

Gemcitabine, oxaliplatin \& cetuximab

A multicenter Phase II study of gemcitabine $\left(1000 \mathrm{mg} / \mathrm{m}^{2}\right.$ on day 1) plus oxaliplatin
(100 $\mathrm{mg} / \mathrm{m}^{2}$ on day 2, every 2 weeks; GEMOX) combined with cetuximab $\left(400 \mathrm{mg} / \mathrm{m}^{2}\right.$ initially then $250 \mathrm{mg} / \mathrm{m}^{2}$ weekly) in 45 untreated patients with advanced stage HCC reported a median progression-free and overall survival of 4.7 and 9.5 months, respectively, with a 1-year survival rate of $40 \%$. This combination appeared to have manageable toxicity [48].

\section{Everolimus \& sorafenib}

A Phase I study, designed to determine the maximum tolerated dose of everolimus given in combination with sorafenib at $400 \mathrm{mg}$ twice daily in patients with advanced Child-Pugh A, HCC who were naive to systemic therapy, demonstrated an inability to achieve a biologically effective everolimus concentration at the maximum tolerated dose (which was $2.5 \mathrm{mg}$ once daily) and precluded Phase II study of the combination [49].

In the SHARP study, baseline ANG 2 and VEGF plasma concentrations independently predicted survival as did clinical variables such as macroscopic vascular invasion, ECOG performance status, baseline $\alpha$-fetoprotein and alkaline phosphatase concentrations. None of the ten plasma biomarkers implicated in the pathogenesis of HCC measured in the SHARP study significantly predicted response to sorafenib [50]. Baseline tumor characteristics could not be studied for predictors of sorafenib benefit as tumor banking was not a priority in that study.

The adverse events that were more common in the SHARP study, sorafenib treatment group, were diarrhea, weight loss and hand-foot skin reaction (HFSR) and were mainly mild-to-moderate in severity. A randomized controlled Phase II study of the prophylactic effect of urea-based cream on the HFSR associated with sorafenib in advanced HCC concluded that compared with best supportive care, prophylactic topical use of a urea-based cream appeared to be effective in preventing and/or delaying the incidence of HFSR with the median time to the first HFSR event being 84 days in the prophylactic urea-based group and 34 days in the best supportive care group (excluding urea-based creams) [51].

\section{Second-line systemic therapies in HCC}

A necessity exists for second-line therapy in patients with advanced HCC who progress after sorafenib. In the clinic setting, there is a patient population clearly well enough to receive 
further therapies if available. Multiple pathways are involved in HCC oncogenesis, proliferation and survival and many novel agents have been investigated and are currently being investigated in second-line advanced HCC and some will now be reviewed.

\section{- Brivanib}

In a Phase II study, brivanib, a selective dual inhibitor of FGF and VEGF signaling was administered as second-line therapy at a dose of $800 \mathrm{mg}$ once daily in 46 patients with advanced HCC. The tumor response rate was $4.3 \%$, disease control rate was $45.7 \%$, median overall survival was 9.79 months and median time to progression was 2.7 months [52]. However, a Phase III randomized trial comparing brivanib versus placebo as second-line therapy in 395 patients with advanced HCC did not meet its primary end point as there was no difference in overall survival seen (8.2 vs 9.4 months). This occurred despite an improvement in response rate (12 vs $2 \%$ in placebo) and progression-free survival (4.1 vs 2.7 months) [53].

\section{- Regorafenib}

A multicenter Phase II safety study of the multikinase inhibitor regorafenib at $160 \mathrm{mg}$ once daily in cycles of 3 weeks on/1 week off treatment, in 36 patients with HCC (Child-Pugh A) that had progressed following first-line sorafenib, reported acceptable tolerability and evidence of anti-tumor activity with disease control seen in 26 patients (one partial response, 25 stable disease). Median time to progression was 4.3 months, and median overall survival was 13.8 months [54]. A Phase III trial of regorafenib after sorafenib in patients with advanced HCC is currently recruiting participants [107].

\section{- Sunitinib}

Sunitinib provided only modest anti-tumor activity in a small study of 11 patients (Child-Pugh A/B) with advanced HCC who had progressed on first-line sorafenib treatment [55].

\section{- Axitinib}

There are two ongoing Phase II trials of axitinib (potent multitarget tyrosine kinase inhibitor of VEGFR1-3, PDGF and c-KIT), in secondline treatment of patients with advanced HCC $[108,109]$. Interim analysis from one of these trials has reported that axitinib is tolerated and has shown preliminary efficacy in a VEGF pretreated Child-Pugh A/B7, HCC patient population [56].

\section{- Everolimus}

A Phase I/II study of the mTOR inhibitor, everolimus, in 28 patients with histologically confirmed measurable advanced HCC with zero to two prior regimens reported that everolimus was well tolerated and demonstrated preliminary anti-tumor activity in patients with advanced HCC most of whom had prior systemic treatment. In this study, $10 \mathrm{mg} /$ day was defined as the Phase II dosage [57]. A randomized Phase III, double-blind, placebo-controlled, multicenter study is currently evaluating the efficacy and safety of everolimus (RAD001) in adult patients with advanced Child-Pugh A, HCC after failure of sorafenib treatment in the EVOLVE-1 study [110]. A press release by Novartis (Basel, Switzerland) in August 2013 regarding this study has revealed that everolimus did not extend overall survival compared with placebo, and therefore Novartis will not proceed with regulatory filings of everolimus in this indication [110].

\section{- Temsirolimus}

A Phase II study of the mTOR inhibitor, temsirolimus, as second-line therapy in patients with advanced, unresectable HCC (Child-Pugh A/B) is also currently ongoing with the primary outcome measure being to determine the proportion of patients who are progression free at 3 months [111].

\section{- Tivantinib}

Tivantinib (ARQ 197) is an oral, selective c-MET inhibitor. A randomized controlled Phase II trial of tivantinib versus placebo in Child-Pugh A patients with HCC who failed one systemic therapy reported that tivantinib improved time to progression, the primary study end point, most significantly in patients with high MET expression (22 out of 107 patients enrolled in study; a factor found to be associated with poor prognosis). The median time to progression was extended from 6.1 weeks with placebo to 11.7 weeks with tivantinib (HR: 0.43; $\mathrm{p}=0.03)$. This MET high subgroup demonstrated a 3.4 month improvement in overall survival with tivantinib compared with placebo (7.2 vs 3.8 months; HR: 0.38; $\mathrm{p}=0.01$ ). No improvements in time to progression or overall survival were observed with tivantinib in patients with low MET expression tumors. No overall 
survival benefit was observed in the overall patient population [58]. The original tivantinib dose of $360 \mathrm{mg}$ twice daily orally was reduced to $240 \mathrm{mg}$ twice daily following an unexpectedly high incidence of neutropenia. A Phase III randomized, double blind study of tivantinib in preselected subjects with tumor MET diagnostic-high inoperable HCC, treated with one prior systemic therapy is currently ongoing [112]. We look forward to the results of this progressive trial design.

\section{- Cabozantinib}

Results from a Phase II randomized trial also demonstrated activity of cabozantinib (XL184, an oral potent inhibitor of MET and VEGFR2). At a dose of $100 \mathrm{mg}$ once daily in 41 Child-Pugh A HCC patients, a median progression-free survival of 4.2 months was reported. The overall disease control rate (partial response and stable disease) at week 12 was 68\% (Asian subgroup $73 \%$ ). The safety profile was comparable to that of other VEGFR tyrosine kinase inhibitors [59]. A Phase III randomized, double-blind, controlled study of cabozantinib (XL184) versus placebo in subjects with HCC who have received prior sorafenib is ongoing [113].

Combination systemic therapy in second-line treatment of advanced HCC

- Bevacizumab \& erlotinib

A Phase II study of the combination of bevacizumab at $10 \mathrm{mg} / \mathrm{kg}$ every 2 weeks and erlotinib $150 \mathrm{mg}$ daily for a maximum of six cycles in patients with advanced Child-Pugh A, HCC with sorafenib-refractory disease, concluded that the combination was well tolerated, but had no activity in an unselected sorafenib refractory advanced HCC population and was halted in the first stage after ten patients were recruited [60].

\section{- Gemcitabine \& oxaliplatin}

A report on 18 consecutive patients with advanced HCC (Child-Pugh A/B) pretreated with sorafenib who received gemcitabine $\left(1000 \mathrm{mg} / \mathrm{m}^{2}\right)$ and oxaliplatin $\left(100 \mathrm{mg} / \mathrm{m}^{2}\right)$ every 14 days as second-line treatment, had a median progression-free survival of 3.2 months (95\% CI: 2.3-3.9) and a median overall survival of 4.7 months (95\% CI: 3.8-8.1 months). While this is not clinically meaningful, it is noteworthy that overall survival was significantly longer in patients without sarcopenia (10.0 months [95\% CI: 7.0-13.8] vs 3.0 months [95\% CI: 2.5-3.9]; $\mathrm{p}<0.001)$ and in patients with an ECOG performance status $<2$ (8.1 months [95\% CI: 7.0-13.8] vs 3.8 months [95\% CI: 2.5-3.9]; $\mathrm{p}=0.017$ ) as it suggests these are the patients most likely to benefit from therapies beyond sorafenib. The most frequent toxicities were thrombocytopenia (grade 2-4; 38.9\%) and peripheral neuropathy (grade 2-3; 38.9\%) [61]. Further studies are required to confirm these findings.

\section{Conclusion}

Despite the discovery of the beneficial role of the multikinase inhibitor, sorafenib, in the systemic treatment of advanced HCC, there is continued necessity for development of effective agents with minimal toxicity for application to all stages of this devastating disease. Some ongoing clinical trials have been discussed and some may eventually provide additional interventional systemic strategy ideas, with further data possibly stimulating exploration of combinations of targeted therapy and/or more conventional cytotoxic drugs that may improve both progression-free and overall survival. It is becoming abundantly clear that demonstrating a superiority over sorafenib is very challenging and, in addition, no novel agent has shown clear benefit post sorafenib. The interpretation of efficacy signals seen in Phase II studies have not held up with proven benefit in the Phase III setting. Assumptions and clinical benchmarks must be carefully considered when designing new trials. Undoubtedly, a greater understanding of signaling pathways in HCC and the identification of biomarkers of response and resistance will be imperative to advance systemic options and move the field towards patientdirected therapy becoming a reality. Therefore, translational studies need to be the standard and acceptance of tumor and tissue banking in this patient population is long overdue. Approval of sorafenib as a systemic agent in the treatment of advanced HCC, was significant progress following a long period of hopelessness and purveyed evidence that systemic treatment has utility as an adjunct and primary therapy in HCC. Improving on the modest survival advantage offered by sorafenib with other related agents has proven to be surprisingly challenging.

\section{Future perspective}

$\mathrm{HCC}$ is a heterogeneous disease with various possible etiologies, and whether the underlying presence of hepatitis B or C will impact on development or response to therapeutic 
agents in the future requires prospective evaluation, as does treatment options for patients with Child-Pugh B, HCC and those recurring after transplant. Other compounds targeting pathways in hepatocarcinogenesis, such as the $\mathrm{PI} 3 \mathrm{~K} / \mathrm{Akt} / \mathrm{mTOR}$ pathway, IGF and its receptor, as well as the $\mathrm{Wnt} / \beta$-catenin pathway are under early stage evaluation in HCC and may enrich the systemic therapeutic field in the future. Oncolytic viruses and active immunotherapeutics may also hold promise for the treatment of HCC in the future. JX-594 (Pexa-Vec, Jennerex Biotherapeutics, CA, USA) is an oncolytic and immunotherapeutic vaccinia virus. A randomized Phase II dose-finding trial in 30 patients with advanced HCC has reported that following infusion of JX-594 into liver tumors, favorable radiological and survival results were observed [62], and has prompted further clinical trials [114,115].

Other important practice changing developments are likely to be in the area of biomarker discovery with their subsequent diagnostic, prognostic and predictive ability possibly changing the landscape of hepatic oncology therapeutics into the future. Given the heterogeneity of the patient population and of HCC, this is absolutely necessary to advance the field. In addition, the importance of continued education of at risk patients to prevent the onset of HCC cannot be overemphasized.

\section{Financial \& competing interests disclosure}

JJ Knox has received research support from Pfizer and Bayer Healthcare Pharmaceuticals, as well as consulting fees from ArQule Pharmaceuticals, Exelixis and Therapure Biopharma. The authors have no other relevant affliations or financial involvement with any organization or entity with a financial interest in or financial conflict with the subject matter or materials discussed in the manuscript apart from those disclosed.

No writing assistance was utilized in the production of this manuscript.

\section{References}

Papers of special note have been highlighted as:

- of interest

-. of considerable interest

1 Ferlay J, Shin HR, Bray F, Forman D, Mathers C, Parkin DM. Estimates of wordwide burden of cancer in 2008: GLOBOCAN 2008. Int. J. Cancer 127(12), 2893-2917 (2010).

2 Bruix J, Sherman M; Practice Guidelines Committee, American Association for the Study of Liver Diseases. Management of hepatocellular carcinoma. Hepatology 42(5), 1208-1236 (2005).

3 Llovet JM, Bruix J. Systematic review of randomized trials for unresectable hepatocellular carcinoma: chemoembolization improves survival. Hepatology 37(2), 429-442 (2003).

4 Llovet JM, Ricci S, Mazzaferro V et al. Sorafenib in advanced hepatocellular carcinoma. N. Engl. J. Med. 359(4), 378-390 (2008).

- Median survival and time to radiologic progression was nearly 3 months longer for patients with advanced hepatocellular carcinoma (HCC) treated with sorafenib compared with patients administered with placebo.

5 Cervello M, McCubrey JA, Cusimano A, Lampiasis N, Azzolina A, Montalto G. Targeted therapy for hepatocellular carcinoma: novel agents on the horizon. Oncotarget 3(3), 236-260 (2012).
6 Goyal L, Muzumdar MD, Zhu AX. Targeting the HGF/c-MET pathway in hepatocellular carcinoma. Clin. Cancer Res. 19(9), 2310-2318 (2013).

7 Wang J, He XD, Yao N, Liang WJ, Zhang YC. A meta-analysis of adjuvant therapy after potentially curative treatment for hepatocellular carcinoma. Can. J. Gastroenterol. 27(6), 351-363 (2013).

8 Lencioni R, Crocetti L. Local-regional treatment of hepatocellular carcinoma. Radiology 262(1), 43-58 (2012).

9 Lammer J, Malagari K, Vogl T et al. Prospective randomized study of doxorubicineluting-bead embolization in the treatment of hepatocellular carcinoma: results of the PRECISION V study. Cardiovasc. Interv. Radiol. 33(1), 41-52 (2010).

- Transarterial chemoembolization, with drug-eluting beads and doxorubicin, is safe and effective in the treatment of HCC and offers a benefit to patients with more advanced disease.

10 Bujold A, Massey CA, Kim JJ et al. Sequential Phase I and II trials of stereotactic body radiotherapy for locally advanced hepatocellular carcinoma. J. Clin. Oncol. 31(13), 1631-1639 (2013).

11 Hong TS. Radiotherapy for hepatocellular carcinoma with tumor vascular thrombus: ready for prime time? J. Clin. Oncol. 31(13), 1619-1620 (2013).

12 Reig M, Burrel M, Bruix J. Treatment of hepatocellular carcinoma with radioembolization: gathering assumptions for trial design. J. Vasc. Interv. Radiol. 24(8), 1197-1199 (2013).

13 Vouche M, Kulik L, Atassi R et al. Radiological-pathological analysis of WHO, RECIST, EASL, mRECIST and DWI: imaging analysis from a prospective randomized trial of $Y 90 \pm$ sorafenib. Hepatology doi:10.1002/hep.26487 (2013) (Epub ahead of print).

14 Lencioni R, Llovet JM, Han G et al. Sorafenib or placebo in combination with transarterial chemoembolization (TACE) with doxorubicin-eluting beads (DEBDOX) for intermediate-stage hepatocellular carcinoma (HCC): Phase II, randomized, double-blind SPACE trial. J. Clin. Oncol. 30(Suppl. 4), Abstract LBA154 (2012).

15 Llovet JM, Bru C, Bruix J. Prognosis of hepatocellular carcinoma: the BCLC staging classification. Semin. Liver Dis. 19(3), 329-338 (1999).

16 Lencioni R. Management of hepatocellular carcinoma with transarterial chemoembolization in the era of systemic targeted therapy. Crit. Rev. Oncol. Hematol. 83(2), 216-224 (2012).

17 Kudo M, Imanaka K, Chida N et al. Phase III study of sorafenib after transarterial chemoembolization in Japanese and Korean patients with unresectable hepatocellular carcinoma. Eur. J. Cancer 47(14), 2117-2127 (2011).

18 Maeda N, Osuga K, Higashihara $\mathrm{H}$ et al. Transarterial chemoembolization with 
cisplatin as second-line treatment for hepatocellular carcinoma unresponsive to chemoembolization with epirubicin-lipiodol emulsion. Cardiovasc. Interv. Radiol. 35(1), 82-89 (2012).

19 Buijs M, Reyes DK, Pawlik TM et al. Phase 2 trial of concurrent bevacizumab and transhepatic arterial chemoembolization in patients with unresectable hepatocellular carcinoma. Cancer 119(5), 1042-1049 (2013).

20 Inaba Y, Kanai F, Aramaki T et al. A randomised Phase II study of TSU-68 in patients with hepatocellular carcinoma treated by transarterial chemoembolization. Eur. J. Cancer 49(13), 2832-2840 (2013).

21 Bruix J, Raoul JL, Sherman M et al. Efficacy and safety of sorafenib in patients with advanced hepatocellular carcinoma: subanalyses of a Phase III trial. J. Hepatol. 57(4), 821-829 (2012).

22 Cheng AL, Guan Z, Chen Z et al. Efficacy and safety of sorafenib in patients with advanced hepatocellular carcinoma according to baseline status: subset analyses of the Phase III sorafenib Asia-Pacific trial. Eur. J. Cancer 48(10), 1452-1465 (2012).

23 Cheng AL, Kang YK, Chen Z et al. Efficacy and safety of sorafenib in patients in the Asia-Pacific region with advanced hepatocellular carcinoma: a Phase III randomised, double-blind, placebo-controlled trial. Lancet Oncol. 10(1), 25-34 (2009).

24 Cheng A, Kang Y, Lin D et al. Phase III trial of sunitinib (Su) versus sorafenib (So) in advanced hepatocellular carcinoma (HCC). J. Clin. Oncol. 29(Suppl.), Abstract 4000 (2011).

25 Lencioni R, Kudo M, Ye SL et al. First interim analysis of the GIDEON ((Global Investigation of therapeutic decisions in hepatocellular carcinoma and of its treatment with sorafeNib) non-interventional study. Int. J. Clin. Pract. 66(7), 675-683 (2012).

26 Kostner AH, Sorensen M, Olesen RK, Gronbaek H, Lassen U, Ladekarl M. Sorafenib in advanced hepatocellular carcinoma: a nationwide retrospective study of efficacy and tolerability. Sci. World J. 2013, 931972 (2013).

27 Cainap C, Qin S, Huang WT et al. Phase III trial of linifanib versus sorafenib in patients with advanced hepatocellular carcinoma (HCC). J. Clin. Oncol. 30(Suppl. 34), Abstract 249 (2012).

28 Johnson PJ, Qin S, Park JW et al. Brivanib versus sorafenib as first-line therapy in patients with unresectable, advanced hepatocellular carcinoma: results from the randomized Phase III BRISK-FL study. J. Clin. Oncol. 31(28), 3517-3524 (2013).
29 Zhu AX, Finn RS, Mulcahy MF et al. A Phase II study of ramucirumab as first-line monotherapy in patients (pts) with advanced hepatocellular carcinoma (HCC). J. Clin. Oncol. 28(Suppl.), Abstract 4083 (2010).

30 Alberts SR, Fitch TR, Kim GP et al. Cediranib (AZD2171) in patients with advanced hepatocellular carcinoma: a Phase II North Central Cancer Treatment Group Clinical trial. Am. J. Clin. Oncol. 35(4), 329-333 (2012).

31 Kanai F, Yoshida H, Tateishi R et al. A Phase I/II trial of the oral antiangiogenic agent TSU-68 in patients with advanced hepatocellular carcinoma. Cancer Chemother. Pharmacol. 67(2), 315-324 (2011).

32 Mitsunaga S, Ikeda M, Ueno $\mathrm{H}$ et al. Phase I/II study of lenvatinib (E7080), a multitargeted tyrosine kinase inhibitor, in patients (pts) with advanced hepatocellular carcinoma (HCC): Phase I results. J. Clin. Oncol. 30(Suppl. 34), Abstract 231 (2012).

33 Yau T, Chen PJ, Chan P et al. Phase I dose-finding study of pazopanib in hepatocellular carcinoma: evaluation of early efficacy, pharmacokinetics, and pharmacodynamics. Clin. Cancer Res. 17(21), 6914-6923 (2011).

34 Zhu AX, Stuart K, Blaszkowsky LS et al. Phase 2 study of cetuximab in patients with advanced hepatocellular carcinoma. Cancer 110(3), 581-589 (2007).

35 Ramanathan RK, Belani CP, Singh DA et al. A Phase II study of lapatinib in patients with advanced biliary tree and hepatocellular cancer. Cancer Chemother. Pharmacol. 64(4), 777-783 (2009).

36 Bekaii-Saab T, Markowitz J, Prescott N et al. A multi-institutional Phase II study of the efficacy and tolerability of lapatinib in patients with advanced hepatocellular carcinomas. Clin. Cancer Res. 15(18), 5895-5901 (2009).

37 O’Neil BH, Goff LW, Kauh JS et al. Phase II study of the mitogen-activated protein kinase $1 / 2$ inhibitor selumetinib in patients with advanced hepatocellular carcinoma. J. Clin. Oncol. 29(17), 2350-2356 (2011).

38 Yeo W, Chung HC, Chan SL et al. Epigenetic therapy using belinostat for patients with unresectable hepatocellular carcinoma: a multicenter Phase I/II study with biomarker and pharmacokinetic analysis of tumors from patients in the Mayo Phase II consortium and the cancer therapeutics research group. J. Clin. Oncol. 30(27), 3361-3367 (2012).

39 Shiah HS, Chen CY, Dai CY et al. Randomised clinical trial: comparison of two everolimus dosing schedules in patients with advanced hepatocellular carcinoma. Aliment. Pharmacol. Ther. 37(1), 62-73 (2013).

40 Abou-Alfa GK, Johnson P, Knox JJ et al. Doxorubicin plus sorafenib vs doxorubicin alone in patients with advanced hepatocellular carcinoma, a randomized trial. JAMA 304(19), 2154-2160 (2010).

- Treatment of patients with advanced HCC with sorafenib plus doxorubicin compared with doxorubicin monotherapy resulted in greater median time to progression, overall survival and progression-free survival, but is not yet indicated for routine clinical use.

41 Qin S, Bai Y, Lim HY et al. Randomized, multicenter, open-label study of oxaliplatin plus fluorouracil/leucovorin versus doxorubicin as palliative chemotherapy in patients with advanced hepatocellular carcinoma from Asia. J. Clin. Oncol. 31(28), 3501-3508 (2013).

42 Thomas MB, Morris JS, Chadha R et al. Phase II trial of the combination of bevacizumab and erlotinib in patients who have advanced hepatocellular carcinoma. J. Clin. Oncol. 27(6), 843-850 (2009).

43 Kaseb AO, Garrett-Mayer E, Morris JS et al. Efficacy of bevacizumab plus erlotinib for advanced hepatocellular carcinoma and predictors of outcome: final results of a Phase II trial. Oncology 82(2), 67-74 (2012).

$44 \mathrm{Hsu} \mathrm{CH}$, Kang YK, Yang TS et al. Bevacizumab with erlotinib as first-line therapy in Asian patients with advanced hepatocellular carcinaom: a multicenter Phase II study. Oncology 85(1), 44-52 (2013).

45 Govindarajan R, Siegel E, Makhoul I, Williamson S. Bevacizumab and erlotinib in previously untreated inoperable and metastatic hepatocellular carcinoma. Am. J. Clin. Oncol. 36(3), 254-257 (2013).

46 Knox JJ, Qin R, Strosberg JR et al. A Phase II trial of temsirolimus (TEM) and bevacizumab $(\mathrm{BEV})$ in patients with advanced hepatocellular carcinoma (HCC). J. Clin. Oncol. 30(Suppl.), Abstract 4099 (2012).

47 Sanoff HK, Bernard S, Goldberg RM et al. Phase II study of capecitabine, oxaliplatin, and cetuximab for advanced hepatocellular carcinoma. Gastrointest. Cancer Res. 4(3), 78-83 (2011).

48 Asnacios A, Fartoux L, Romano O et al. Gemcitabine plus oxaliplatin (GEMOX) combined with cetuximab in patients with progressive advanced stage hepatocellular carcinoma: results of a multicenter Phase 2 study. Cancer 112(12), 2733-2739 (2008). 
49 Finn RS, Poon RT, Yau T et al. Phase I study investigating everolimus combined with sorafenib in patients with advanced hepatocellular carcinoma. J. Hepatol. doi:10.1016/j.jhep.2013.07.029 (2013) (Epub ahead of print).

50 Llovet JM, Pena CE, Lathia CD et al. Plasma biomarkers as predictors of outcome in patients with advanced hepatocellular carcinoma. Clin. Cancer Res. 18(8), 2290-2300 (2012).

51 Ren Z, Zhu K, Kang H et al. A randomized controlled Phase II study of the prophylactic effect of urea-based cream on the hand-foot skin reaction associated with sorafenib in advanced hepatocellular carcinoma. J. Clin. Oncol. 30(Suppl.), Abstract 4008 (2012).

- Prophylactic topical use of a urea-based cream appears to be effective in preventing and/or delaying the incidence of hand-foot skin reaction associated with sorafenib treatment in patients with advanced HCC.

52 Finn RS, Kang YK, Mulcahy M et al. Phase II, open-label study of brivanib as second-line therapy in patients with advanced hepatocellular carcinoma. Clin. Cancer Res. 18(7), 2090-2098 (2012).

53 Llovet JM, Decaens T, Raoul JL et al. Brivanib in patients with advanced hepatocellular carcinoma who were intolerant to sorafenib or for whom sorafenib failed: results from the randomized Phase III BRISK-PS study. J. Clin. Oncol. 31(28), 3509-3516 (2013).

54 Bruix J, Tak WY, Gasbarrini A et al. Regorafenib as second-line therapy for intermediate or advanced hepatocellular carcinoma: multicentre, open-label, Phase II safety study. Eur. J. Cancer 49(16), 3412-3419 (2013).

55 Worns MA, Schuchmann M, Duber C et al. Sunitinib in patients with advanced hepatocellular carcinoma after progression under sorafenib treatment. Oncology 79(1-2), 85-92 (2010).

56 McNamara MG, Horgan AM, Aspinall A et al. A Phase II trial of second-line axitinib following prior antiangiogenic therapy in advanced hepatocellular carcinoma (HCC). J. Clin. Oncol. 30(Suppl. 34), Abstract 314 (2012).

57 Zhu AX, Abrams TA, Miksad R et al. Phase $1 / 2$ study of everolimus in advanced hepatocellular carcinoma. Cancer 117(22), 5094-5102 (2011).

58 Rimassa L, Porta C, Borbath I et al. Tivantinib (ARQ 197) versus placebo in patients (pts) with hepatocellular carcinoma (HCC) who failed one systemic therapy: results of a randomized controlled Phase II trial (RCT). J. Clin. Oncol. 30(Suppl.), Abstract 4006 (2012).

-. Tivantinib significantly benefited second-line HCC patients compared with placebo in time to progression, disease control rate and progression-free survival, with preliminary overall survival trends, especially in Met $^{+}$patients.

59 Verslype C, Cohn AL, Kelley RK et al. Activity of cabozantinib (XL184) in hepatocellular carcinoma: results from a Phase II randomized discontinuation trial (RDT). J. Clin. Oncol. 30(Suppl.) Abstract 4007 (2012).

- Cabozantinib treatment (potent inhibitor of MET and VEGF receptor 2) exhibits activity in HCC patients regardless of prior sorafenib treatment.

60 Yau T, Wong H, Chan P et al. Phase II study of bevacizumab and erlotinib in the treatment of advanced hepatocellular carcinoma patients with sorafenib-refractory disease. Invest. New Drugs 30(6), 2384-2390 (2012).

61 Mir O, Coriat R, Boudou-Rouquette P et al. Gemcitabine and oxaliplatin as second-line treatment in patients with hepatocellular carcinoma pre-treated with sorafenib. Med. Oncol. 29(4), 2793-2799 (2012).

62 Heo J, Reid T, Ruo L et al. Randomized dose-finding clinical trial of oncolytic immunotherapeutic vaccinia JX-594 in liver cancer. Nat. Med. 19(3), 329-336 (2013).

\section{- Websites}

101 Sorafenib as Adjuvant Treatment in the Prevention Of Recurrence of Hepatocellular Carcinoma (STORM). http://clinicaltrials.gov/ct2/show/ NCT00692770

102 Efficacy Evaluation of TheraSphere in Patients With Inoperable Liver Cancer (STOP-HCC).

http://clinicaltrials.gov/ct2/show/ NCT01556490

103 Sorafenib Tosylate With or Without Stereotactic Body Radiation Therapy in Treating Patients With Liver Cancer. http://clinicaltrials.gov/ct2/show/ NCT01730937

104 A Study of Ramucirumab (IMC-1121B) Drug Product (DP) and Best Supportive Care (BSC) Versus Placebo and BSC as 2nd-Line Treatment in Patients With Hepatocellular Carcinoma After 1st-Line Therapy With Sorafenib (REACH). http://clinicaltrials.gov/ct2/show/ NCT01140347
105 A Multicenter, Open-Label, Phase 3 Trial to Compare the Efficacy and Safety of Lenvatinib (E7080) Versus Sorafenib in First-Line Treatment of Subjects With Unresectable Hepatocellular Carcinoma (REFLECT).

http://clinicaltrials.gov/ct2/show/ NCT01761266

106 Sorafenib Tosylate With or Without Doxorubicin Hydrochloride in Treating Patients With Locally Advanced or Metastatic Liver Cancer.

http://clinicaltrials.gov/ct2/show/ NCT01015833

107 Study of Regorafenib After Sorafenib in Patients With Hepatocellular Carcinoma (RESORCE).

http://clinicaltrials.gov/ct2/show/ NCT01774344

108 Study of Axitinib in Patients With Unresectable Hepatocellular Carcinoma (AXITINIB).

http://clinicaltrials.gov/ct2/show/ NCT01334112

109 Axitinib as Second-line Treatment for Advanced Hepatocellular Carcinoma. http://clinicaltrials.gov/ct2/show/ NCT01273662

110 Global Study Looking at the Combination of RAD001 Plus Best Supportive Care (BSC) and Placebo Plus BSC to Treat Patients With Advanced Hepatocellular Carcinoma (EVOLVE-1). http://clinicaltrials.gov/ct2/show/ NCT01035229

111 Temsirolimus as Second-line Therapy in HCC.

http://clinicaltrials.gov/ct2/show/ NCT01567930

112 Study of Tivantinib in Subjects With Inoperable Hepatocellular Carcinoma Who Have Been Treated With One Prior Therapy (METIV-HCC). http://clinicaltrials.gov/ct2/show/ NCT01755767

113 Study of Cabozantinib (XL184) vs Placebo in Subjects With Hepatocellular Carcinoma Who Have Received Prior Sorafenib (CELESTIAL). http://clinicaltrials.gov/ct2/show/ NCT01908426

114 A Phase 2a Study of Modified Vaccinia Virus to Treat Sorafenib-naive Advanced Liver Cancer (FLASH). http://clinicaltrials.gov/ct2/show/ NCT01636284

115 A Phase 2b Study of Modified Vaccinia Virus to Treat Patients Advanced Liver Cancer Who Failed Sorafenib (TRAVERSE). 
MANAGEMENT PERSPECTIVE

http://clinicaltrials.gov/ct2/show/ NCT01387555

116 Transarterial Chemoembolization Using Doxorubicin Beads With or Without Sorafenib Tosylate in Treating Patients With Liver Cancer That Cannot Be Removed By Surgery.

http://clinicaltrials.gov/ct2/show/ NCT01324076
117 Chemoembolization With or Without Sorafenib Tosylate in Treating Patients With Liver Cancer That Cannot Be Removed By Surgery.

http://clinicaltrials.gov/ct2/show/

NCT01004978

118 Orantinib In Combination With

Transcatheter Arterial Chemoembolization In
Patients With Unresectable Hepatocellular Carcinoma (ORIENTAL).

http://clinicaltrials.gov/ct2/show/

NCT01465464

119 Phase III Trans-Arterial Chemo-Embolization (TACE) Adjuvant HCC (BRISK TA).

http://clinicaltrials.gov/ct2/show/

NCT00908752 